\title{
NON-TRAUMATIC MEDIASTINAL EMPHYSEMA IN CHILDHOOD
}

\author{
BY \\ WILLIAM H. PATTERSON and JOHN FAWCITT \\ From Booth Hall Hospital, Manchester
}

(RECEIVED FOR PUBLICATION MAY 19, 1954)

This condition has been described as occurring in both adults and children and is probably uncommon rather than rare. There are few recorded cases in the British paediatric literature, but in discussion of the subject with colleagues occasional instances in children have been mentioned.

In the absence of the associated subcutaneous emphysema the mediastinal collection of air is likely to be overlooked clinically, but the increased resort to radiography in acute chest illness may well reveal that it is not so very uncommon as a complication.

After a perusal of the literature it was considered of interest that four patients-one infant and three children-had been encountered in a short period of time. In these patients the condition had not come within either the spontaneous or the traumatic groups but was secondary to recognized pulmonary illness.

\section{Case Reports}

Case 1. J.G., a girl, aged 10 months, was admitted to hospital in the third week of pertussis because of a convulsion and developed a measles rash the day following. Eleven days later a widespread subcutaneous emphysema was seen which involved the whole of the upper thorax and extended to the face, neck and scalp. The infant was cyanosed, dyspnoeic and ill, with an exhausting cough before this emphysema was noted, and its onset had not been preceded by any noticeable deterioration in her condition.

Radiographs confirmed the presence of mediastinal and subcutaneous emphysema associated with areas of lobular collapse and consolidation in both lung fields. There was no pneumothorax.

The emphysema took three weeks to absorb completely. The infant eventually recovered after a stormy illness which included a second bronchopneumonic episode, without complications.

The complication of measles in the third week of a severe pertussis infection provided the requisite conditions for the development of either a pneumothorax or mediastinal emphysema. The forced expiratory explosions of the whooping cough and the inflammatory pulmonary lesions of the measles caused what was probably, from the degree and extent of the emphysema, a considerable pulmonary rent.

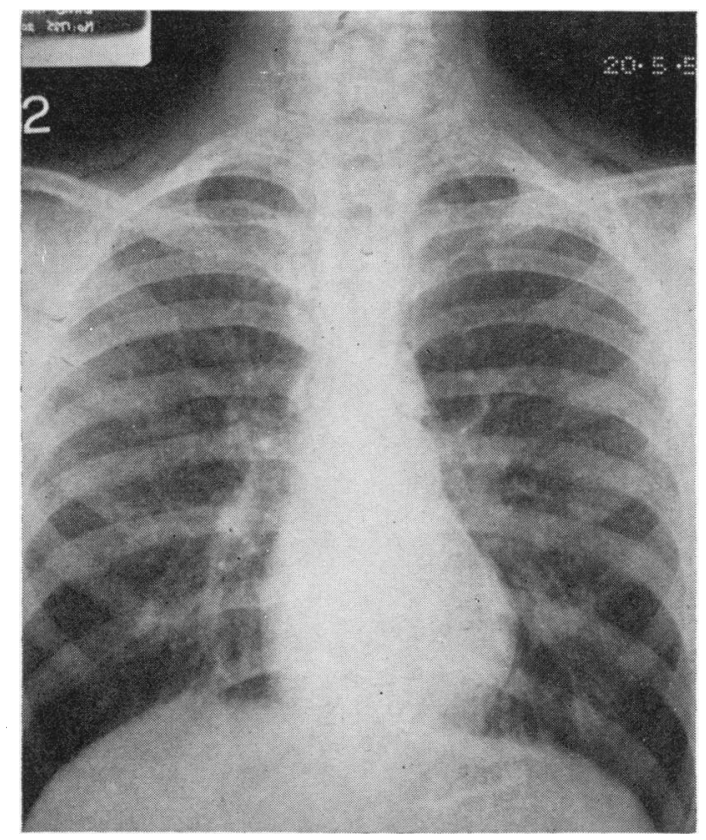

FIG. 1.-Radiograph showing double contour of the left border of the heart and subcutaneous emphysema of the neck.

Case 2. D.J., a boy, aged 13 years, had suffered from asthma since infancy. Two days before we saw him he had had a severe asthmatic episode with much retching and vomiting. He complained of a sharp substernal pain of brief duration during the attack. He saw his doctor next day because of swelling of the neck which was recognized as subcutaneous emphysema and he was referred to hospital.

Clinically the boy was not acutely ill. He had subcutaneous emphysema mainly confined to the supraclavicular regions. The superficial area of cardiac 
dullness was diminished and the heart sounds were distant. There was general hyper-resonance to percussion and the breath sounds were diminished throughout the lung fields.

Radiologically there were emphysematous changes in both lung fields with a double contour round the left border of the heart. Oblique and lateral views showed the air in the mediastinum to extend into the root of the neck, and there was evidence of air in the superficial tissues of the supraclavicular regions (Fig. 1).

The lungs were emphysematous due to longstanding asthma, and an acute asthmatic attack with straining and vomiting was followed by mediastinal and subcutaneous emphysema.

Case 3. J.B., a girl, aged $2 \frac{1}{2}$ years, was admitted to hospital on the third day of an acute respiratory illness with a temperature of $104^{\circ} \mathrm{F}$. Constitutional symptoms were predominant and cough was not noticeably troublesome. A clinical diagnosis of pneumonia in the right lung was made and the child was nursed in an oxygen tent and given antibiotics.

The day after admission a crepitant swelling appeared in the suprasternal region (Fig. 2). This increased in size during that day and then gradually subsided.

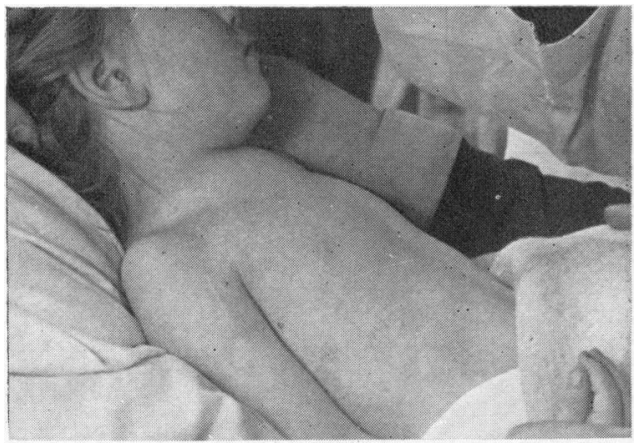

Fig. 2.-Photograph showing distension of the suprasternal region by subcutaneous air.

Radiographs taken two days after the appearance of the subcutaneous emphysema showed patchy consolidation of the left lower lobe, collapse of the right middle lobe and mediastinal and subcutaneous emphysema; the latter was mainly in the upper thorax and neck.

The child was apyrexial by the ninth day of illness and made an uncomplicated recovery.

The sequence was that of a bronchopneumonia complicated by lobar collapse with pulmonary emphysema, air escaping into the interstitial portion of the lung with consequent mediastinal and subcutaneous emphysema.

Case 4. M.W., a girl, aged 5 years, was very well up to 48 hours before admission to hospital. Her illness began without fever or coryzal symptoms but the child developed a persistent cough which was very troublesome at night but less so during the day when she was not confined to bed. There was no constitutional upset.

On the day of admission (the third day of symptoms) she was 'a little weary' but was not dyspnoeic or cyanosed; she appeared however to be afraid to take a very deep breath.

At this stage the family doctor was consulted, detected subcutaneous emphysema, and sent the patient to hospital.

On admission the relevant clinical findings were of a not acutely ill child. The temperature was $99^{\circ} \mathrm{F}$; pulse 112 , and there was no dyspnoea, cough or cyanosis. There was subcutaneous emphysema of the upper thorax, including the axillae, and extending into the cervical region. The loud crackling of the emphysema obscured the breath sounds. It was not possible to localize the apex beat and the heart sounds were distant.

In 24 hours the subcutaneous emphysema had spread considerably and had reached the parotid area on the right side. Thereafter it gradually disappeared and was undetectable clinically by the sixth day after admission.

Of the investigations carried out the only relevant findings were a slightly raised blood sedimentation rate (18 mm. in one hour by micro method) and a total leucocyte count of 16,600 per c.mm. The Mantoux test ( 1 in 1,000 old tuberculin) was negative.

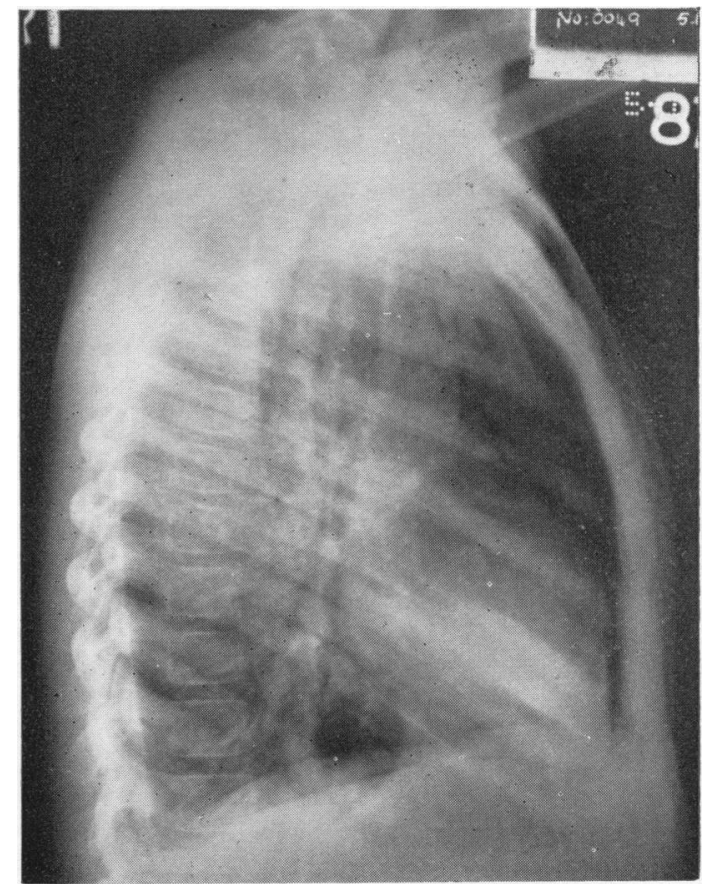

FIG, 3-Air shown outlining mediastinal structures as well as some superficial to the sternum. Right middle lobe collapse is also evident. 
Radiographs (Figs. 3 and 4) taken on the third day following the appearance of subcutaneous emphysema showed collapse of the right middle lobe with marked emphysema outlining the structures of the anterior mediastinum particularly well in the lateral view of the chest. This projection also demonstrated subcutaneous emphysema lying anteriorly to the sternum. A posteroanterior view of the chest showed air in the superficial

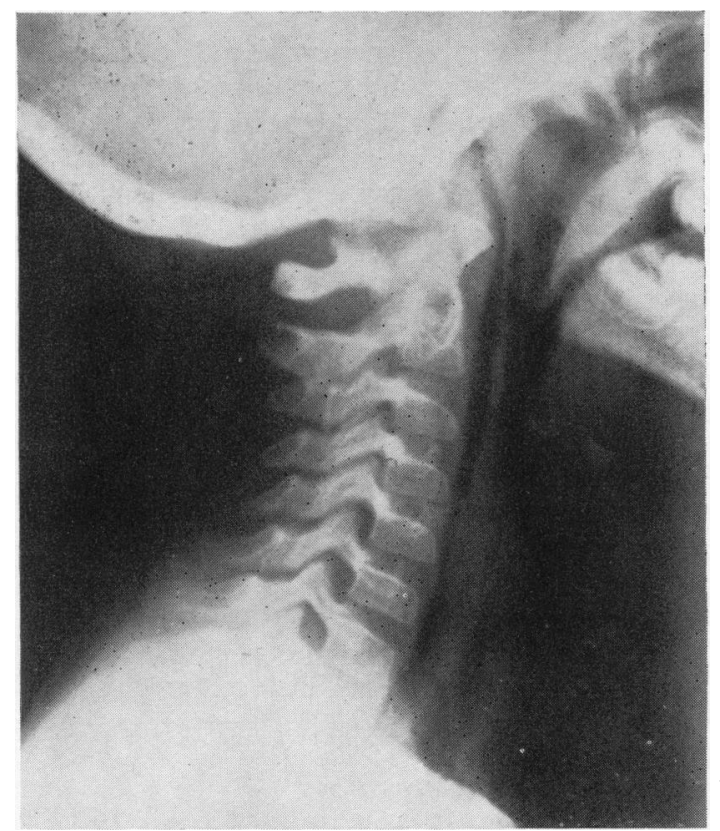

Fig. 4.-A lateral radiograph shows air in soft tissue planes of the neck.

tissues of the neck, whilst a lateral view showed the air to have extended into the pre-cervical fascia.

Two weeks from the onset of the illness the chest radiograph was within the bounds of normal.

The sequence of events was a probable tracheitis with a persistent cough producing collapse of the right middle lobe and subsequent pulmonary, mediastinal and subcutaneous emphysema.

\section{Radiological Features}

The precursor of non-traumatic mediastinal emphysema is almost invariably pulmonary interstitial emphysema, and since the radiological features of this condition have recently been very fully described by Herrnheiser and Whitehead (1953) they will not now be discussed.

When a minor degree of mediastinal emphysema is present this extra-pulmonary air may not be observed in the routine postero-anterior view of the chest, and oblique or lateral projections are necessary to demonstrate it. These smaller amounts of air are generally shown as streaky shadows in the anterior and posterior mediastina. They accentuate the outlines of the vessels and organs in those areas, more particularly the anterior border of the heart, the thymus in children, and the aorta and the great vessels, extending even up into the root of the neck. In one of our cases the apex of the heart was clearly outlined, and the air accentuated its diaphragmatic border. An associated pneumothorax is not common with this degree of pneumomediastinum.

When a larger amount of air has found its way into the mediastinum it is generally readily seen in the postero-anterior radiograph as a double contour round a portion of the cardiac shadow. It often tracks up into the soft tissues of the neck, either in the compartments of the great vessels, behind the sternum anteriorly, or posteriorly into the space between the prevertebral fascia of the neck and the pharynx. A pneumothorax, which, if present, is almost invariably left sided, may cloak these radiological appearances to some degree.

In the very severe cases where the mediastinal air is present in large amounts, the cardiac shadow may appear to be smaller than normal and to be displaced. The sternum may appear to be bulged forward.

The air may be shown radiologically to be extensively spread in the superficial tissues, and this extension may relieve the pressure on the heart and the great vessels.

Careful note should be taken of any radiological changes in the lung fields of these cases, particularly findings suggestive of asthma and lobar or lobular collapse.

It is considered that the proportion of cases in which the air is seen in the superficial tissues is higher in children than in adults.

\section{Theories of Aetiology}

Pneumomediastinum was described before the days of Laennec, and Müller in $\mathbf{1 8 8 8}$ gave a graphic account of the clinical signs. It is, however, on the work of Macklin (1939) and Hamman (1945) that the modern conception of non-traumatic mediastinal emphysema really rests.

Macklin, following experimental work on cats, suggested that hyperinflation of the alveoli bordering on blood vessels tended to build up an intraalveolar pressure greater than that within these vessels, causing a rupture of the alveolar bases and escape of air into the perivascular sheaths. These tiny bubbles of air passed down the sheaths towards the hilum, and, tending to coalesce, formed blebs of air in the hilar region whence they burst into the mediastinum. In addition Macklin considered that there were extensions of air into the contiguous 
connective tissue formations in the region of the hila. This mechanism not only caused a degree of vascular stasis due to the air in the perivascular sheaths occluding the vessels, but reduced the respiratory excursion by 'splinting' the lung tissue. This conception of the mechanism of the formation of non-traumatic pneumomediastinum has now been widely accepted.

When the air reaches the mediastinum there are three possible courses open to it. (1) It may remain within the mediastinum and build up a pressure which will cause partial occlusion of the great vessels and consequent cardiac embarrassment and dyspnoea. (2) In approximately a third of the cases a pneumothorax occurs, which Macklin considers may be caused by air from the mediastinum, though he does not consider that the air from a pneumothorax can travel in the opposite direction. (3) The air may track up into the superficial and deep tissue planes in the neck and into the superficial tissues of the thorax and trunk, thus markedly relieving the pressure in the mediastinum.

The condition may be associated with any lesion causing localized atelectatic changes in the lungs which could be surrounded by an area of hyperinflated lung tissue. It is considered that an element of congenital weakness must be present in this lung tissue as well.

\section{Clinical Features}

The occurrence of mediastinal emphysema, with or without an associated pneumothorax, is of course well known to the thoracic surgeon. Its appearance following tracheotomy operations has often been described and is readily explained, and nonoperative pulmonary trauma from missiles and blast has provided a number of cases in recent years.

Another group, spontaneous mediastinal emphysema, has been so called because it occurs in previously healthy individuals in whom no underlying pathological condition can be demonstrated. This group is important since, being uncomplicated by trauma or associated pathological cause, its symptoms and signs are those of mediastinal emphysema per se. These are the sudden onset of chest pain and dyspnoea with an absence of constitutional symptoms. In about half these cases there is also an associated spontaneous pneumothorax.

Clinically, about a quarter of these spontaneous cases have subcutaneous emphysema. The area of cardiac dullness is diminished, but the characteristic feature is a peculiar sound over the cardiac region. It is known as Hamman's sign and is variously described as 'crunching', 'crackling', 'clicking', 'like the rattling of dried peas on taut canvas' or 'the crinkling of a newspaper'. It is heard throughout the respiratory cycle and may even be audible to the patient himself.

Our four cases, being children, were unable to describe their symptoms adequately, though the boy of 13 did complain of sharp chest pain. All had some underlying illness the symptoms of which obscured the onset of the mediastinal emphysema. It seems likely that if the mediastinal air is able to escape before much intra-mediastinal pressure is built up, then acute subjective symptoms may be minimal or absent. The subcutaneous spread of air is an escape way.

In two of our cases it was possible to estimate that they were in no way inconvenienced by the air in the mediastinum. One patient walked quite a distance to the Out-patient Department, and the other was referred to hospital because her doctor recognized the presence of something unusual and not because she was seriously ill.

In none of our cases was Hamman's sign detected. Previous authors have emphasized that it is by no means always present and is seldom detected in infants and young children. Draper (1948), however, found the sign in $90 \%$ of the spontaneous variety, and we suspect that the high incidence of associated left-sided pneumothorax in those cases may be responsible for helping to bring out the characteristic sounds.

\section{Treatment}

We have no comment to offer on treatment.

Our patients required therapy only for the underlying pathological condition. From our small experience we might agree with the dictum that mediastinal emphysema 'is a relatively harmless condition'.

\section{Summary}

The relevant clinical and radiological features of four cases of non-traumatic mediastinal emphysema are presented.

The aetiological and mechanistic theories are discussed.

We should like to acknowledge with thanks the courtesy of Dr. S. K. Guthrie of the Duchess of York Hospital for Babies and Dr. D. C. Liddle of Monsall Hospital, Manchester, for allowing us to use cases which had been under their care; Dr. J. Wraith, for the loan of the radiographs, and Mr. Ward for the photograph.

\section{REFERENCES}

Draper, A. J. (1948). Amer. J. Med., 5, 59.

Hamman, L. (1945). J. Amer. med. Ass., 128, 1.

Herrnheiser, G. and Whitehead, J. P. (1953). Brit. J. Radiol., 26, 519.

Macklin, C. C. (1939). Arch. intern. Med., 64, 913. 